\title{
EFICIÊNCIA OPERACIONAL DA SERRA DE FITA NO DESDOBRO DE EUCALIPTO
}

\author{
Douglas Edson Carvalho ${ }^{1}$, Márcio Pereira da Rocha ${ }^{2}$, Ricardo Jorge Klitzke ${ }^{2}$, Pedro Henrique Gonzalez de Cademartori ${ }^{2}$ \\ 1 Programa de Pós-graduação em Engenharia Florestal, Universidade Federal do Paraná, 80201-170, Curitiba, Brasil. \\ 2 Departamento de Engenharia e Tecnologia Florestal, Universidade Federal do Paraná, 80201-170, Curitiba, Brasil.
}

*E-mail:_douglasedsoncarvalho@gmail.com

\section{RESUMO}

O presente estudo teve como objetivo avaliar a eficiência operacional da máquina principal (serra de fita) no desdobro de toras de Eucalyptus spp.. As toras foram divididas em quatro classes diamétricas (classe 1:21,0 a 30,9 cm; classe 2: 31,0 a 40,9 cm; classe 3: 41,0 a 50,9 cm; classe 4: > 51,0 cm) cada classe com três comprimentos de tora $(3,60 \mathrm{~m} ; 4,10 \mathrm{~m} ; 5,20 \mathrm{~m})$. As duas classes diamétricas inferiores ( 1 e 2) foram submetidas ao modelo de desdobro tangencial alternado e as toras das duas classes diamétricas superiores ( $3 \mathrm{e}$ 4) foram submetidas ao modelo de desdobro radial. Em cada classe diamétrica as toras foram avaliadas quanto à conicidade, à influência do comprimento e ao diâmetro da tora na eficiência operacional. Para cada modelo de desdobro foram geradas equações para estimar a eficiência operacional. O acréscimo em comprimento e diâmetro das toras para cada modelo de desdobro proporcionou aumento da eficiência operacional média. As estratificações dimensionais e as baixas conicidades das toras associadas ao modelo de desdobro apresentam resultados de eficiência operacional média na máquina principal entre 17,70 e $34,57 \mathrm{~m}^{3}$.operário ${ }^{-1}$.turno ${ }^{-1}$. A variável volume das toras apresenta correlação positiva e direta com eficiência operacional.

Palavras-chave: Madeira serrada. Eucalypus. Modelo de desdobro. Eficácia

\section{Introdução}

A matéria-prima madeira é utilizada amplamente no setor industrial para confecção de diversos produtos obtidos de madeira serrada ou de seus subprodutos. Entretanto, para o avanço do setor madeireiro, novos métodos são necessários para elevar o parâmetro eficiência operacional de produção e, consequentemente, pode-se defini-lo como fator que determina a evolução do processo produtivo.

Beltrame et al. [1] salientam a necessidade de estudos com o gênero Eucalyptus, qualificando as madeiras a fim de obter um produto sólido. Conforme relatório IBÁ [2], entre os vários setores produtivos da economia brasileira, o setor de florestas plantadas é o que oferece maior potencial de apoio para a construção sustentável, cujas empresas estão comprometidas em utilizar melhores práticas socioambientais, o que implica em maximizar a eficiência e o rendimento dos processos produtivos.

Para a avaliação de comportamento e desempenho de uma serraria diversos parâmetros podem ser aplicados, dentre eles, citam-se o rendimento e a eficiência operacional, os quais demonstram com clareza se as atividades da serraria estão atingindo o desempenho desejado [3].
Marchesan et al. [4] e Heinrich [5] afirmam que, para a continuidade e permanência ativa de empresas do setor madeireiro no mercado, a eficiência operacional e econômica na transformação da matéria-prima tora em madeira serrada é primordial para o sucesso das empresas deste ramo.

Vital [6] e Manhiça et al. [7] alegam que o modelo de corte utilizado altera o rendimento e eficiência da serraria, além da qualidade da madeira serrada. Além dos parâmetros citados é importante ressaltar os demais fatores que podem interferir na eficiência operacional de máquinas e implementos de serrarias, dentre eles o comprimento, o diâmetro e a conicidade das toras usadas no processo de desdobro, além das variações de dimensões dos produtos obtidos e a experiência do operador.

O modelo de desdobro afeta a qualidade do produto final e a produtividade, que por sua vez apresenta maior eficiência operacional no desdobro tangencial em comparação ao desdobro radial [6, 8-9]. Entretanto, o uso de modelos específicos para cada classe diamétrica pode minimizar esta desigualdade de eficiência entre diferentes tipos de desdobro.

Segundo Batista et al. [10], a análise da eficiência por parte de grandes empresas do setor madeireiro não é aplicada

TECNO-LÓGICA, Santa Cruz do Sul, v. 23, n. 1, p. 36-41, jan./jul. 2019 
devido ao nível de automação das mesmas. Este fato está associado com o nível de otimização destas empresas. Contudo, a realidade brasileira conta com um número significativo de serrarias consideradas de pequeno e médio porte e segundo Latorraca [11], este tipo de empresas apresenta controle automatizado mínimo, tornando o parâmetro eficiência operacional fundamental para planejamentos de produção e tomada de decisões.

Baseado nas evidências supracitadas a respeito dos baixos investimentos em tecnologia, na maioria das serrarias brasileiras e na importância de manutenção da qualidade do processo de beneficiamento de madeira, este estudo teve como objetivo avaliar a eficiência operacional da serra de fita no desdobro de toras de Eucalyptus spp.. Para fins de investigação da eficiência operacional, foram avaliados três comprimentos de tora e quatro classes diamétricas, divididas em dois modelos de desdobro.

\section{Materiais e métodos}

\subsection{Local de estudo e coleta de dados}

Os dados para o estudo foram obtidos em uma serraria localizada no município de Campina Grande do Sul, no Estado do Paraná, região metropolitana de Curitiba, coordenadas $25^{\circ} 20^{\prime} 42.6^{\prime \prime} \mathrm{S}$ e $49^{\circ} 03^{\prime} 25.0^{\prime \prime} \mathrm{W}$. A empresa é considerada de médio porte e atua no mercado interno brasileiro atendendo ao setor moveleiro e, principalmente, o setor de construção civil, utilizando espécies de Eucalyptus provenientes de plantios nos Estados do Paraná, São Paulo e Santa Catarina, classificadas somente quanto aos comprimentos das toras (3,60 m; 4,10 m; 5,20 m).

Para condução do estudo foram utilizadas 168 toras divididas em quatro classes diamétricas (Tabela 1) e posteriormente foram aplicados dois modelos de desdobro, denominados desdobro tangencial alternado para as classes diamétricas 1 e 2 (inferiores) e desdobro radial para as classes diamétricas 3 e 4 (superiores), conforme modelos de Carvalho [12]. Em ambos os planos de corte foram obtidas pranchas com espessura de $50 \mathrm{~mm}$, larguras e comprimentos variados.

Tabela 1. Distribuição das classes diamétricas, diâmetros e comprimentos de toras de Eucalyptus spp. nos modelos de desdobro aplicados em cada classe.

\begin{tabular}{cccc}
\hline $\begin{array}{c}\text { Classe } \\
\text { diamétrica }\end{array}$ & $\begin{array}{c}\text { Diâmetro das } \\
\text { toras }(\mathbf{c m})\end{array}$ & $\begin{array}{c}\text { Comprimento } \\
\text { das toras }(\mathbf{m})\end{array}$ & $\begin{array}{c}\text { Modelo } \\
\text { de } \\
\text { desdobro }\end{array}$ \\
\hline Classe 1 & $21,0-30,9$ & $3,60 / 4,10 / 5,20$ & Tangencial \\
Classe 2 & $31,0-40,9$ & $3,60 / 4,10 / 5,20$ & alternado \\
\cline { 4 - 4 } Classe 3 & $41,0-50,9$ & $3,60 / 4,10 / 5,20$ & Radial \\
Classe 4 & $>51,0$ & $3,60 / 4,10 / 5,20$ & \\
\hline
\end{tabular}

\subsection{Determinação das variáveis}

Foram realizadas medições de comprimento $(\mathrm{m}) \mathrm{e}$ circunferência $(\mathrm{mm})$ na ponta fina e na ponta grossa das toras com auxílio de fita métrica e transformada em diâmetro $(\mathrm{cm})$ para posterior cálculo do volume de cada tora (Equação 1, adaptada de Smalian) e determinação do volume médio por comprimento e classe diamétrica (Tabela 2).

$$
\text { V Tora }=\frac{\pi}{40000} \times\left(\frac{\mathrm{D} 1+\mathrm{D} 2}{2}\right)^{2} \times \mathrm{C}
$$

em que:

V Tora $=$ volume da tora $\left(\mathrm{m}^{3}\right)$;

D1= diâmetro da ponta fina $(\mathrm{cm})$;

D2 = diâmetro da ponta grossa $(\mathrm{cm})$;

$\mathrm{C}=$ comprimento da tora $(\mathrm{m})$.

Tabela 2. Volume médio $\left(\mathrm{m}^{3}\right)$ das toras de cada comprimento e classe diamétrica.

\begin{tabular}{ccc|cc}
\hline $\begin{array}{c}\text { Modelo de } \\
\text { desdobro }\end{array}$ & \multicolumn{2}{c|}{$\begin{array}{c}\text { Tangencial } \\
\text { alternado }\end{array}$} & \multicolumn{2}{|c}{ Radial } \\
\hline Comp. (m) & Classe $\mathbf{1}$ & $\begin{array}{c}\text { Classe } \\
\mathbf{2}\end{array}$ & Classe 3 & Classe 4 \\
\hline 3,60 & 0,2403 & 0,2977 & 0,5613 & 1,0261 \\
4,10 & 0,2531 & 0,3481 & 0,6155 & 1,1351 \\
5,20 & 0,3291 & 0,4731 & 0,8301 & 1,1481 \\
\hline
\end{tabular}

Para o cálculo da conicidade das toras foi utilizada a equação 2, conforme Scanavaca Jr. e Garcia [13]:

$$
\text { Conic }(\mathrm{cm} / \mathrm{m})=\frac{\mathrm{D} 2-\mathrm{D} 1}{\mathrm{C}}
$$

em que:

Conic $=$ conicidade da tora $(\mathrm{cm} / \mathrm{m})$;

A eficiência operacional da serra fita vertical foi determinada a partir da equação 3, adaptada de Rocha [3], a qual é calculada pelo volume de tora desdobrada em determinado período e o número de operários envolvidos na operação de desdobro.

$$
E f=\frac{480 \times V}{t \times O}
$$

em que:

Ef = eficiência $\left(\mathrm{m}^{3}\right.$ operário $^{-1}$ turno $\left.^{-1}\right)$;

480 = tempo em minutos correspondentes a 08 horas de trabalho, um turno ou dia de trabalho;

$\mathrm{V}=$ volume da tora $\left(\mathrm{m}^{3}\right)$;

$\mathrm{t}=$ tempo em minutos para desdobro do volume de tora;

$\mathrm{O}=$ número de operários que trabalham no processo de desdobro principal.

O tempo considerado a um turno ou dia de trabalho equivale a 08 horas (480 minutos). Este tempo foi multiplicado pelo volume de cada tora serrada, obtido da cubagem das mesmas. O valor obtido foi dividido pela multiplicação no número de 
operários e tempo gasto para desdobro de cada tora. Este tempo era cronometrado para cada tora a partir do momento em que esta era carregada no carro porta-toras até o momento em que a última peça era retirada da serra de fita vertical. No modelo de desdobro tangencial alternado eram necessários três funcionários, enquanto no modelo de desdobro radial quatro funcionários. Com isso, obteve-se a eficiência média operacional para cada classe diamétrica em função dos comprimentos de tora e modelos de desdobro.

Para o desdobro de toras do modelo tangencial alternado foi utilizada uma serra de fita vertical simples com volante de $1.350 \mathrm{~mm}$ de diâmetro. Para o desdobro de toras do modelo radial foi utilizada a serra de fita vertical citada anteriormente para abertura da tora ao meio e posteriormente uma serra de fita vertical simples com volante de $1.100 \mathrm{~mm}$ de diâmetro para o desdobro das duas metades de cada tora.

\subsection{Análise dos dados}

Previamente à análise de variância (ANOVA), para identificar diferenças estatísticas, foi realizado o teste de Skewness e Kurtosis para avaliar a normalidade dos dados. Identificadas diferenças estatísticas, procedeu-se com a comparação múltipla de médias por meio do teste de Tukey $(\mathrm{p} \leq 0,05)$.

Complementarmente, foi calculada a correlação linear de Pearson (r) a fim de verificar a relação existente entre as variáveis: comprimento, diâmetro médio, volume e conicidade da tora com a eficiência operacional da serra de fita para os modelos de desdobro aplicados, com o auxílio de planilha eletrônica.

\section{Resultados e discussões}

\subsection{Conicidade}

Observando a conicidade das toras utilizadas nos modelos de desdobro (Figura 1), todas apresentaram conicidade média considerada baixa, inferior a $3 \mathrm{~cm} \cdot \mathrm{m}^{-1}$, conforme a Norma para Classificação de Toras de Madeira de Folhosas [14], apesar das oscilações observadas.

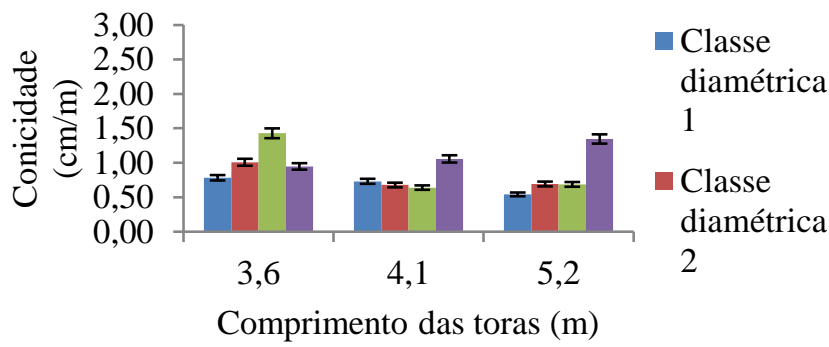

Figura 1. Conicidade das toras utilizadas nos modelos de desdobro distribuídas em comprimentos e classes diamétricas.
Entretanto, valores mais elevados de conicidade influenciam negativamente na eficiência operacional, uma vez que o operador da serra de fita realiza mais movimentos e operações sobre a tora para acomodação, ajuste e corte da mesma.

As toras da menor classe diamétrica e as toras de maior comprimento apresentaram a menor conicidade média em geral. Este comportamento é atribuído à posição em que a tora foi retirada da árvore, porque esse fator está relacionado com a diminuição do diâmetro do tronco, que ocorre da base para a copa da árvore [15]. Vale ressaltar que as toras com valores com conicidade acima da média, como as toras da classe 4 para o corte no plano radial provavelmente são toras da base. Todavia outras variáveis podem influenciar neste parâmetro, como idade e espaçamento do plantio.

\subsection{Eficiência operacional}

No desdobro tangencial alternado (Tabela 3), observamos diferença significativa entre as classes diamétricas. Já em relação aos comprimentos notamos que a elevação deste parâmetro resultou em superioridade da eficiência operacional, apresentando diferença significativa entre as toras de maior comprimento em relação às de menor comprimento.

Tabela 3. Comparativo da eficiência operacional ( $\mathrm{m}^{3} /$ operário/turno) em duas classes diamétricas e três comprimentos de tora no modelo de desdobro tangencial alternado para toras de Eucalyptus spp.

\begin{tabular}{ccc}
\hline Comprimento de & \multicolumn{2}{c}{ Eficiência operacional $\left(\mathbf{m}^{\left.\mathbf{3} . \mathbf{o p}^{\mathbf{- 1}} \cdot \mathbf{t}^{\mathbf{- 1}}\right)}\right.$} \\
\cline { 2 - 3 } tora $(\mathbf{m})$ & Classe $\mathbf{1}$ & Classe $\mathbf{2}$ \\
\hline 3,60 & $27,48(8,91) \mathrm{B} \mathrm{b}$ & $29,72(7,53) \mathrm{A} \mathrm{b}$ \\
4,10 & $26,42(10,99) \mathrm{B} \mathrm{ab}$ & $32,82(7,65) \mathrm{A} \mathrm{a}$ \\
5,20 & $30,94(17,25) \mathrm{B} \mathrm{a}$ & $34,57(12,99) \mathrm{A} \mathrm{a}$ \\
\hline Média & 28,28 & 32,37 \\
\hline
\end{tabular}

Médias seguidas pela mesma letra minúscula na coluna, e pela mesma letra maiúscula na linha, não diferem entre si estatisticamente pelo teste de Tukey a 5\% de probabilidade $(0,01=<\mathrm{p}<0,05)$. Valores entre parênteses referem-se ao coeficiente de variação (\%) das amostras.

Utilizando o desdobro radial (Tabela 4), a eficiência operacional apresentou diferença significativa entre as classes diamétricas somente para as toras de 4,10 m, o que está associada a erros práticos operacionais, pois entre os demais comprimentos não se observou este comportamento. 
Tabela 4. Comparativo da eficiência operacional ( $\mathrm{m}^{3} /$ operário/turno) em duas classes diamétricas e três comprimentos de tora no modelo de desdobro radial para toras de Eucalyptus spp..

\begin{tabular}{ccc}
\hline $\begin{array}{c}\text { Comprimento de } \\
\text { tora }(\mathbf{m})\end{array}$ & \multicolumn{2}{c}{ Eficiência operacional $\left(\mathbf{m}^{\mathbf{3} . \mathbf{o p}^{\mathbf{- 1}} \mathbf{.}} \mathbf{\mathbf { - 1 }}\right)$} \\
\cline { 2 - 3 } & Classe 3 & Classe 4 \\
\hline 3,60 & $18,22(11,68) \mathrm{A} \mathrm{a}$ & $20,32(12,39) \mathrm{A} \mathrm{a}$ \\
4,10 & $17,70(14,23) \mathrm{B} \mathrm{a}$ & $23,10(17,93) \mathrm{A} \mathrm{a}$ \\
5,20 & $18,00(17,22) \mathrm{A} \mathrm{a}$ & $23,81(24,82) \mathrm{A} \mathrm{a}$ \\
\hline Média & 17,97 & 22,41 \\
\hline
\end{tabular}

Médias seguidas pela mesma letra minúscula na coluna, e pela mesma letra maiúscula na linha, não diferem entre si estatisticamente pelo teste de Tukey a 5\% de probabilidade $(0,01=<\mathrm{p}<0,05)$. Valores entre parênteses referem-se ao coeficiente de variação $(\%)$ das amostras.

A eficiência operacional do desdobro principal médio obtido (Tabelas 3 e 4) é considerada satisfatória, estando na faixa de 20 a $50 \mathrm{~m}^{3}$ operário/turno para a maioria dos comprimentos e classes diamétricas, citada como a média obtida por serrarias automatizadas do Brasil, conforme Rocha [3], o que favorece a produtividade da empresa.

Não se enquadram nesta faixa as toras da classe diamétrica 3, reiterando que o modelo de desdobro adotado para o diâmetro destas toras pode não ter sido o mais indicado, retardando o processo, o qual resultou em eficiência inferior as demais classes.

Analisando os modelos de desdobro, verificamos que no modelo de desdobro tangencial alternado há aumento da eficiência com o incremento em diâmetro, o mesmo observado, mas em menor intensidade, com o modelo de desdobro radial, corroborando com Marchesan et al. [4] e Biasi [16] que afirmam ocorrer aumento da eficiência técnica da menor para a maior classe diamétrica.

Em geral os resultados inferiores de eficiência operacional obtido para o modelo de desdobro radial pode ser atribuído ao número de funcionários necessários para as operações, a maior rotatividade da tora para seu ajuste e quantidades de cortes devido aos diâmetros mais elevados, considerando que peças de mesmas dimensões foram produzidas em ambos os modelos de desdobro. Neste contexto, Manhiça et al. [7] (2013) afirmaram que de acordo com o modelo de corte aplicado, o operador pode levar mais tempo para desdobrar a tora, consequentemente afetando a eficiência operacional, o que de fato ocorreu com o modelo de desdobro radial.

As eficiências operacionais do desdobro primário estão acima dos valores obtidos por Batista et al. [10], em que os autores encontraram eficiência média de $5,06 \mathrm{~m}^{3}$ /operário/turno, com toras de Eucalyptus spp. de pequenos diâmetros (10 a $40 \mathrm{~cm})$. Em estudo mais recente, Batista et al. [17] alcançaram eficiência operacional média de $2,77 \mathrm{~m}$ /operário/turno. Estas diferenças observadas entre o presente estudo e os supracitados são atribuídas as diferentes classes diamétricas avaliadas bem como o processo analisado, uma vez que os autores citados analisaram a eficiência de todo o processo, incluindo o desdobro secundário, e este pode ser afetado pelo layout da serraria e treinamento dos operadores.

Marchesan et al. [4], analisando a eficiência no desdobro principal das espécies jatobá (Hymenaea cournaril Hayne), muiracatiara (Astronium lecointei Ducke) e muirapiranga (Brosimum rubescens Taub.), observaram eficiência operacional de 2,25, 2,61 e 2,63 m/operário/turno, respectivamente, sendo inferiores ao presente estudo. Esta inferioridade é devido às características das espécies, pois segundo os autores citados anteriormente, as toras podem apresentar altos índices de defeitos, causados por organismos xilófagos, tortuosidades e a cortes mais lentos realizados em função da densidade do material. Em espécies de Eucalyptus, a incidência destes fatores também pode ser observada, porém em menor escala, minimizando a interferência sobre a eficiência operacional.

\subsection{Correlações entre variáveis}

Para o modelo de desdobro tangencial alternado, a variável volume foi a que apresentou maior correlação com a eficiência operacional (Tabela 5), seguida por comprimento e diâmetro médio, respectivamente.

Tabela 5. Matriz de correlação linear de Pearson entre as variáveis comprimento $(\mathrm{C})$, diâmetro médio $(\mathrm{Dm})$, volume $(\mathrm{V})$ e conicidade $(\mathrm{Cn})$ da tora em relação à eficiência operacional do desdobro primário (Ef) para modelo de desdobro tangencial alternado de Eucalyptus spp..

\begin{tabular}{|c|c|c|c|c|c|}
\hline & $\begin{array}{c}\mathrm{C} \\
(\mathbf{m})\end{array}$ & $\begin{array}{c}\mathrm{Dm} \\
(\mathrm{cm})\end{array}$ & $\begin{array}{c}V \\
\left(\mathrm{~m}^{3}\right)\end{array}$ & $\begin{array}{c}\mathrm{Cn} \\
\left(\mathrm{cm} \mathrm{m}^{-1}\right)\end{array}$ & $\begin{array}{c}\text { Ef } \\
\left(\mathrm{m}^{3} \mathrm{op}^{-1} \mathrm{t}^{-1}\right)\end{array}$ \\
\hline
\end{tabular}

\section{C (m)}

\begin{tabular}{|c|c|c|c|c|c|}
\hline Dm (cm) & 0,17 & - & & & \\
\hline$V\left(\mathbf{m}^{3}\right)$ & 0,75 & 0,77 & - & & \\
\hline $\operatorname{Cn}\left(\mathrm{cm} \mathrm{m}^{-1}\right)$ & $-0,32$ & 0,15 & $-0,12$ & - & \\
\hline Ef $\left(m^{3} o p^{-1} t^{-1}\right)$ & 0,52 & 0,46 & 0,64 & $-0,16$ & - \\
\hline
\end{tabular}

A correlação existente entre essas variáveis se mostrou moderada a forte e positiva em relação à eficiência operacional, ou seja, quanto maior o comprimento, diâmetro médio e volume da tora maior será a eficiência operacional para o modelo de desdobro tangencial. Já a variável conicidade apresentou correlação negativa e fraca (próximo de 0 ) em relação à eficiência operacional para esse modelo de desdobro.

Marchesan et al. [4] (2014), comparando a eficiência operacional da serra de fita no desdobro de três espécies amazônicas de importância para o setor madeireiro, constataram 
correlação considerada pelos autores como moderada $(0,35)$ entre o comprimento da tora e a eficiência, a partir do teste de correlação de Pearson para as três espécies.

Em relação ao modelo de desdobro radial (Tabela 6), a variável volume também foi a mais correlacionada com a eficiência operacional seguida pelo diâmetro médio, ambas com correlação moderada a forte e positiva.

Tabela 6. Matriz de correlação linear de Pearson entre as variáveis comprimento (C), diâmetro médio $(\mathrm{Dm})$, volume $(\mathrm{V})$ e conicidade $(\mathrm{Cn})$ da tora em relação à eficiência operacional (Ef) do desdobro primário para modelo de desdobro radial de Eucalyptus spp..

\begin{tabular}{lccccc}
\hline & $\begin{array}{c}\mathbf{C} \\
(\mathbf{m})\end{array}$ & $\begin{array}{c}\mathbf{D m} \\
(\mathbf{c m})\end{array}$ & $\begin{array}{c}\mathbf{V} \\
\left(\mathbf{m}^{\mathbf{3}}\right)\end{array}$ & $\begin{array}{c}\mathbf{C n} \\
\left(\mathbf{c m ~ m}^{-\mathbf{1}}\right)\end{array}$ & $\begin{array}{c}\mathbf{E f} \\
\left(\mathbf{m}^{\mathbf{3}} \mathbf{o p}^{-\mathbf{1}} \mathbf{t}^{-1}\right)\end{array}$ \\
\hline $\mathbf{C}(\mathbf{m})$ & - & & & & \\
$\mathbf{D m}(\mathbf{c m})$ & $-0,36$ & - & & & \\
$\mathbf{V}\left(\mathbf{m}^{\mathbf{3}}\right)$ & 0,05 & 0,91 & - & & \\
$\mathbf{C n}\left(\mathbf{c m ~ m}^{-1}\right)$ & $-0,10$ & $-0,01$ & $-0,03$ & - & \\
$\mathbf{E f ~}\left(\mathbf{m}^{\mathbf{3}} \mathbf{o p}^{-\mathbf{1}} \mathbf{t}^{-\mathbf{1}}\right)$ & $-0,01$ & 0,52 & 0,59 & 0,10 & - \\
\hline
\end{tabular}

Para esse modelo de desdobro, a variável comprimento apresentou correlação linear fraca e negativa, comportamento contrário ao constatado para o modelo de desdobro tangencial alternado, indicando que para o modelo de desdobro radial, o comprimento não interfere diretamente na eficiência operacional do desdobro na serra de fita.

\section{Conclusões}

O incremento em diâmetro e comprimento das toras proporciona aumento da eficiência operacional.

A maior eficiência operacional foi obtida na classe diamétrica 2, com toras de $5,20 \mathrm{~m}$, equivalendo a $34,57 \mathrm{~m}^{3}$. operário $^{-1}$ turno $^{-1}$, e a menor eficiência operacional foi de 17,70 $\mathrm{m}^{3}$.operário ${ }^{-1}$.turno ${ }^{-1}$ na classe diamétrica 3 com toras de $4,10 \mathrm{~m}$.

O volume da tora é a variável que apresenta melhor correlação positiva com a eficiência operacional nos modelos de desdobro avaliados.

\section{Agradecimentos}

Ao Conselho Nacional de Desenvolvimento Científico e Tecnológico (CNPq) e a Mademape - Indústria Madeireira Ltda.

\section{OPERATIONAL EFFICIENCY OF BAND SAW IN EUCALYPTUS CUTTING}

ABSTRACT: The present study aimed to evaluate the operational efficiency of the main machine (band saw) used for sawing logs of Eucalyptus spp. The logs were divided into four diameter classes (Class 1: 21.0 to $30.9 \mathrm{~cm}$; Class 2: 31.0 to $40.9 \mathrm{~cm}$; Class 3: 41.0 to $50.9 \mathrm{~cm}$; Class $4:>51.0 \mathrm{~cm}$ ), each class with three log lengths $(3.60 \mathrm{~m} ; 4.10 \mathrm{~m} ; 5.20 \mathrm{~m})$. The two lower diameter class were subjected to the alternating tangential cutting model and the logs of the two higher diameter classes were subjected to the radial cutting model. For each cutting model, equations were generated to estimate the operational efficiency. The increase in length and diameter of the logs for each cutting model provided an increase in the average operational efficiency. The dimensional stratifications and the low conicity of the logs associated with the cutting model presented results of average operational efficiency in the main machine between 17.70 and $34.57 \mathrm{~m}^{3}$ worker ${ }^{-1}$ shift $^{-1}$. The volume of the logs presented a positive and direct correlation with operational efficiency.

Keywords: Sawn wood. Eucalyptus. Cutting model. Efficiency.

\section{Referências}

[1] BELTRAME, R.; PERES, M. L. da; DELUCIS, R. de A.; FRETIAS, D. L. de; GATTO, D. A.; HASELEIN, C. R. Tensões de crescimento longitudinais e propriedades mecânicas da madeira de clones de Eucalyptus spp. Matéria (Rio J.), Vol. 20, n. 4, p. 1061-1074, 2005. http://dx.doi.org/10.1590/S1517707620150004.0108

[2] INDÚSTRIA BRASILEIRA DE ÁRVORES (IBÁ). Relatório Técnico 2015. São Paulo, 2015.

[3] ROCHA, M. P. da. Técnicas de planejamento em serrarias. Série Didática FUPEF, Curitiba, n. 02/01, 121 p, 2002.

[4] MARCHESAN, R.; ROCHA, M. P. da; SILVA, J. B. da; KLITZKE, R. J. Eficiência técnica no desdobro principal de toras de três espécies tropicais. FLORESTA, Curitiba, PR, Vol. 44, n. 4, p. 629-636, 2014.

[5] HEINRICH, D. Simulação da produção de madeira serrada. 2010. 157p. (Dissertação de mestrado). Universidade Federal do Rio Grande do Sul.

[6] VITAL, B. R. Planejamento e operação de serrarias. Viçosa, MG: UFV, 2008.

[7] MANHIÇA, A. A; ROCHA, M. P. da; TIMOFEICZYK JUNIOR, R. Eficiência operacional no desdobro de Pinus utilizando modelos de corte numa serraria de pequeno porte. Cerne. Vol. 19, n. 2, p.339-346, 2013. http://dx.doi.org/10.1590/S0104-77602013000200019.

[8] ROCHA, M. P. da; TOMASELLI, I. Efeito de modelo de corte nas dimensões de madeira serrada de Eucalyptus grandis e Eucalyptus dunnii. Floresta e Ambiente, Vol. 8, n. 1, p 94-103, 2001.

[9] ROCHA, M.P.; TOMASELLI, I. Efeito do modelo de desdobro na qualidade da madeira serrada de Eucalyptus grandis e Eucalyptus dunnii. Cerne, Vol. 8, n. 2, p.70-83, 2002. 
[10] BATISTA, D. C.; SILVA, J. G. M.; CORTELETTI, R. B. Desempenho de uma serraria com base na eficiência e na amostragem do trabalho. Floresta e Ambiente, Vol. 20, n. 2, p. 271 - 280, 2013.

[11] LATORRACA, J. V. F. Processamento mecânico da madeira. Seropédica: Universidade Federal Rural do Rio de Janeiro, 2004. 116 p.

[12] CARVALHO, D. E. Melhoria no desdobro em uma serraria de eucalipto para madeira destinada a construção. 83 p. Dissertação (Mestrado em Engenharia Florestal) - Universidade Federal do Paraná, Curitiba, 2016.

[13] SCANAVACA JÚNIOR, L; GARCIA, J. N.; Rendimento em madeira serrada de Eucalyptus urophylla. Scientia Forestalis, Vol. 63, n. 1, p. 32-43, 2003.

[14] INSTITUTO BRASILEIRO DE DESENVOLVIMENTO FLORESTAL (IBDF). Norma para medição e classificação de toras de madeira de folhosas. Brasília, 1984.

[15] VALÉRIO, A. F.; WATZLAWICK, L. F.; SANTOS, R. T. dos; BRANDELEIRO, C.; KHOELER, H. S. Quantificação de resíduos e rendimento no desdobro de Araucaria angustifolia (bertol.) O. Kuntze. Floresta, Vol. 37, n. 3, p. 387-398, 2007.

[16] BIASI, C. P. Rendimento em madeira serrada, geração de resíduos e eficiência no desdobro de três espécies tropicais. 61 p. Dissertação (Mestrado em Engenharia Florestal) - Universidade Federal do Paraná, Curitiba, 2005.

[17] BATISTA, D. C.; SILVA, J. G. M. da; ANDRADE, W. S. de P.; VIDAURRE, G. B. Desempenho operacional de uma serraria de pequeno porte do município de Alegre, Espírito Santo. Floresta, Vol. 45, n. 3, p. 487-496, 2015. DOI: 10.5380/rf.v45i3.34441 